Appl. Set-Valued Anal. Optim. 3 (2021), No. 2, pp. 149-164

Available online at http://asvao.biemdas.com

https://doi.org/10.23952/asvao.3.2021.2.02

\title{
WEAK APPROXIMATE SOLUTIONS TO QUASI-VARIATIONAL INEQUALITIES: APPLICATION TO SOCIAL NASH EQUILIBRIA
}

\author{
M. AIT MANSOUR ${ }^{1, *}$, J. LAHRACHE ${ }^{2}$, N. ZIANE ${ }^{2}$ \\ ${ }^{1}$ Département de Physiques, LPFAS, Faculté Polydisciplinaire, Safi, Université Cadi Ayyad, Morocco \\ ${ }^{2}$ Département de Mathématiques, Faculté des Sciences, Université Chouaib Doukkali, B.P 20, El Jadida Morocco
}

\begin{abstract}
In this paper, we introduce and prove the existence of the auxiliary solutions of a weak approximate type to quasi-variational inequalities, (QVI), by using the assumptions of minimal character without recourse to the usual semicontinuity and monotonicity conditions. The concept of weak solutions is linked to a new version of weak approximate normal cones to a closed and convex subset which we support by some examples. Moreover, under a mild additional regularity condition, we establish the convergence of those auxiliary solutions to exact ones wherein a qualitative stability result of approximate fixed points is a key ingredient. Our main result is discussed by an application to Social Nash Equilibria for which we obtain weak approximate solutions under weaker assumptions.
\end{abstract}

Keywords. Regularization; Quasi-variational inequality; Weak approximate normal cone; Weak approximate solutions; Social Nash Equilibria.

\section{INTRODUCTION}

In [1], Ait Mansour, Durea and Théra defined a lower semicontinuous regularization for a not necessarily lower semicontinuous set-valued map. It was clearly shown in [1] that this mathematical concept of regularization is valuable in its own by proving a wide connection to interesting problems such as fixed points of set-valued maps, variational inequalities and differential inclusions. Recently, in [2], Ait Mansour, Lahrache and Ziane justified once more the interest of this regularization by investigating quasi-variational inequalities, (QVI) for short, defined by constraints given by a set valued map $\mathbb{K}$ and a set-valued operator $T$. Indeed, they presented the existence of strong $\alpha$-approximate solutions for every $\alpha>0$ without any continuity or monotonicity condition on $T$, where the key idea is to regularize the operator $T$, leading henceforth to a notion of strong approximate normal cone to a closed and convex subset, which generates in turn solutions of a strong approximate type.

In this paper, we involve the regularization rather on the closed convex hull of a slightly modified version of the solution map considered in [2]. In this respect, we introduce a new definition of the just quoted normal cone in a weak format for which concrete examples are provided. On

${ }^{*}$ Corresponding author.

E-mail addresses: ait.mansour.mohamed@gmail.com (M. Ait Mansour), jaafarlahrache@yahoo.fr (J. Lahrache), zianenour@gmail.com (N. Ziane).

Received July 14, 2020; Accepted September 22, 2020.

(C)2021 Applied Set-Valued Analysis and Optimization 
the other hand, the link to a new weak $\alpha$-approximate solutions to quasi-variational inequalities is shown. The existence of this type of weak solutions is thereafter proved without the monotonicity and semicontinuity conditions on the map $T$. Moreover, under a mild additional regularity assumption, we establish the convergence of these weak solutions to exact ones by means of a qualitative stability result of approximate fixed points.

To introduce the (QVI) problem, let $\mathscr{K}$ be a nonempty, compact and convex subset of a Banach space $X$ whose dual, norm and duality pairing are respectively denoted by $X^{*},\|\cdot\|$ and $\langle.,$.$\rangle . The norm in X^{*}$ will also be denoted by $\|$.$\| . The convex hull of a set A$ will be denoted by convA. The feasibility set-valued map will be denoted by $\mathbb{K}: \mathscr{K} \rightrightarrows \mathscr{K}$. It will be assumed to have nonempty, closed and convex values. For a closed convex subset $D$ of $X, N_{D}(\bar{x})$ will stand for the normal cone to $D$ at a point $\bar{x} \in D$, which is given by

$$
N_{D}(\bar{x})=\left\{x^{*} \in X^{*} \mid\left\langle x^{*}, x-\bar{x}\right\rangle \leq 0, \forall x \in D\right\} .
$$

Given a set-valued operator $T: X \rightrightarrows X^{*}$, the corresponding set-valued quasi-variational inequality problem, $\mathrm{QVI}(T, \mathbb{K})$, is defined as follows: Find $\bar{x} \in \mathscr{K}$ such that $\bar{x} \in \mathbb{K}(\bar{x})$ and

$$
0 \in T(\bar{x})+N_{\mathbb{K}(\bar{x})}(\bar{x}) .
$$

This inequality is satisfied if and only if there exists some $x^{*} \in T(\bar{x})$ such that

$$
-x^{*} \in N_{\mathbb{K}(\bar{x})}(\bar{x}) .
$$

Equivalently, using the assumptions on $\mathbb{K}, \mathrm{QVI}(T, \mathbb{K})$ means that

$$
\left\langle x^{*}, y-\bar{x}\right\rangle \geq 0, \forall y \in \mathbb{K}(\bar{x}) .
$$

As emphasized in the Joly-Mosco's scheme [3], problem (QVI) can be treated by analyzing the related (generated) family of variational inequalities $\operatorname{VI}(T, \mathbb{K}(x))_{x \in \mathscr{K}}$ : For all $x \in \mathscr{K}$, find $y(x):=y \in \mathbb{K}(x)$ and $y^{*} \in T(y)$ such that

$$
\left\langle y^{*}, z-y\right\rangle \geq 0, \forall z \in \mathbb{K}(x) .
$$

In this paper, we remark that problem (QVI) is also related to the family of inequalities $\operatorname{VI}(T(x), \mathbb{K}(x))_{x \in \mathscr{K}}$ defined by: For all $x \in \mathscr{K}$, find $y(x):=y \in \mathbb{K}(x)$ and $y^{*} \in T(x)$ such that

$$
\left\langle y^{*}, z-y\right\rangle \geq 0, \forall z \in \mathbb{K}(x) .
$$

Then, by introducing the following auxiliary solution map (slightly modified if compared with the one of [2]) $S: \mathscr{K} \rightrightarrows \mathscr{K}$ defined by for each $x \in \mathscr{K}$

$$
S(x)=\left\{y \in \mathbb{K}(x) \mid \exists y^{*} \in T(x):\left\langle y^{*}, z-y\right\rangle \geq 0, \forall z \in \mathbb{K}(x)\right\},
$$

we observe that the solutions to $\mathrm{QVI}(T, \mathbb{K})$ coincide with the fixed points of $S$. Visibly, $S$ has nonempty values since for all $x \in \mathscr{K}$, and a given $y^{*} \in T(x)$, a minimum point of the continuous map $z \longmapsto\left\langle y^{*}, z\right\rangle$ over the compact $\mathbb{K}(x)$ belongs necessarily to $S(x)$.

This fixed point scheme, very known in the literature (see, for instance, [3, 4]), makes use of classical fixed point theorems such as the Kakutani's theorem and its variants, which requires the upper semicontinuity of the solution map $S$ in addition to the convexity of its values. These properties may not be easy to check sometimes, and they always require the monotonicity and continuity type conditions on $T$. Therefore, our purpose in this paper is to consider approximate versions of the original problem. In this spirit, Ait Mansour, Lahrache and Ziane [2] introduced 
two kinds of approximation of the normal cone to a closed convex subset $D \subset X$ at a point $\bar{x}$ as follows:

Definition 1.1. Let $D$ be a nonempty, closed and convex subset of $X$. For every $\alpha>0$,

(1) the strong $\alpha$-approximate normal cone to $D$ at a point $\bar{x} \in D$, denoted by $s-\mathrm{N}_{D}^{\alpha}(\bar{x})$, is defined by

$$
s-\mathrm{N}_{D}^{\alpha}(\bar{x})=\left\{x^{*} \in X^{*} \mid\left\langle x^{*}, x-\bar{x}\right\rangle \leq \alpha\|x-\bar{x}\|, \forall x \in D\right\}
$$

(see $[5$, p. 6]);

(2) the weak $\alpha$-approximate normal cone to $D$ at a point $\bar{x} \in D$, denoted by $w-\mathrm{N}_{D}^{\alpha}(\bar{x})$, is defined by

$$
w-\mathrm{N}_{D}^{\alpha}(\bar{x})=\left\{x^{*} \in X^{*} \mid\left\langle x^{*}, x-\bar{x}\right\rangle \leq \alpha\left\|x^{*}\right\|, \forall x \in D\right\} .
$$

The weak approximate solutions, to QVI, close to $w-\mathrm{N}_{D}^{\alpha}$ are introduced below (see Definition 2.1). For the strong approximate format for the normal cone, as mentioned above, the corresponding solutions to QVI were established in [2] under weaker assumptions. The aim of the present paper is to extend the results of [2] to the weak format by using the assumptions of minimal character again. As underlined earlier, the crucial step of [2] applies the lower semicontinuous regularization in the sense of [1] upon the operator $T$ while the impact of the regularization is stressed directly on the solution map $S$ defined above on the basis of Mosco and Joly's scheme [3]. This fact brings something new for the treatment of QVI that allows to go into a weak kind of approximate solutions under weak assumptions with a remarkable difference if we compare with the strong solutions obtained in [2].

The paper is organized as follows. In Section 2, we first provide examples of the weak approximate normal cone to a closed convex subset. Then, the corresponding weak solutions to quasi-variational inequalities are fixed. Section 3 recalls set-valued analysis elements necessary for our results. Section 4 is devoted to the main results of this paper concerning the existence of weak approximate solutions to quasi-variational inequalities and their convergence to exact solutions. In Section 5, we discuss an example of the application with respect to the social Nash equilibria modelled by a quasi-variational inequality problem. Finally, we comment our contribution and notice further research questions.

\section{EXAMPLES OF THE WEAK-APPROXIMATE NORMAL CONE AND WEAK APPROXIMATE QUASI-VARIATIONAL INEQUALITIES}

First, observe that the strong approximate normal cone to a closed, convex and nonempty subset set $D\left(s-\mathrm{N}_{D}^{\alpha}(\bar{x})\right)$ is convex, but it is not a cone in general, whereas the weak approximate normal one $w-\mathrm{N}_{D}^{\alpha}(\bar{x})$ is a cone, but not necessarily convex. The classic normal cone $N_{D}(\bar{x})$ is contained in both of them. In the following, we provide some examples of $w-\mathrm{N}_{D}^{\alpha}$.

Example 2.1. A particular example of the weak $\alpha$-approximate normal cone is when $D$ is bounded and $\operatorname{diam}(D):=\sup _{(x, y) \in D^{2}}\|x-y\| \leq \alpha$. In this case, we have $w-\mathrm{N}_{D}^{\alpha}(\bar{x})=X^{*}$. 
Example 2.2. Notice that if $\operatorname{diam}(D)>\alpha$, then we may get the situation where $w-\mathrm{N}_{D}^{\alpha}(\bar{x})=X^{*}$ as shows in the following example. Take $X=\mathbb{R}$ and $\alpha=1$. Consider $D=\{x \in \mathbb{R} \mid-1 \leq x \leq 1\}$, and see that

$$
\begin{aligned}
w-\mathrm{N}_{D}^{1}(0) & =\{s \in \mathbb{R}|\langle s, x\rangle \leq| s \mid, \forall x \in D\} \\
& =\{s \in \mathbb{R}|s x \leq| s \mid, \forall x \in D\} \\
& =\mathbb{R} .
\end{aligned}
$$

Example 2.3. Take $X=\mathbb{R}$ and $\alpha=1$. $D$ is the subset given by

$$
D=\{x \in \mathbb{R} \mid 1 \leq x \leq 3\} .
$$

Observe that

$$
\begin{aligned}
w-\mathrm{N}_{D}^{1}(1) & =\{s \in \mathbb{R}|\langle s, x-1\rangle \leq| s \mid, \forall x \in D\} \\
& =\left\{s \in \mathbb{R}^{-} \mid s(x-1) \leq-s, \forall x \in D\right\} \cup\left\{s \in \mathbb{R}^{+} \mid s(x-1) \leq s, \forall x \in D\right\} \\
& =\left\{s \in \mathbb{R}^{-} \mid s x \leq 0, \forall x \in D\right\} \cup\left\{s \in \mathbb{R}^{+} \mid s(x-2) \leq 0, \forall x \in D\right\} \\
& =\mathbb{R}^{-} \cup\{0\} \\
& =]-\infty, 0] .
\end{aligned}
$$

Example 2.4. Let $X=\mathbb{R}^{2}, \alpha=\frac{12}{10}=1,2$ and $D$ be the subset defined by

$$
D=\left\{(x, y) \in \mathbb{R}^{2} \mid-1 \leq x \leq 1,-1 \leq y \leq 1\right\} .
$$

We consider $w-\mathrm{N}_{D}^{\alpha}$ at the point $(0,0)$ :

$$
w-\mathrm{N}_{D}^{\alpha}(0,0)=\left\{(s, t) \in \mathbb{R}^{2} \mid s x+t y \leq \alpha\|(s, t)\|, \forall(x, y) \in D\right\},
$$

where $\|(s, t)\|=\sqrt{s^{2}+t^{2}}, s, t \in \mathbb{R}$. We make appeal to polar coordinates $s=r \cos (\theta)$ and $t=$ $r \sin (\theta), r>0, \theta \in \mathbb{R}$. After elementary calculus, we find that

$$
w-\mathrm{N}_{D}^{\alpha}(0,0)=C_{1} \cup C_{2} \cup C_{3} \cup C_{4}
$$

with

$$
\begin{aligned}
& C_{1}=\left\{(r \cos \theta, r \sin \theta) \mid(r, \theta) \in\left[0,+\infty\left[\times\left[-\theta_{1}, \theta_{1}\right]\right\} ;\right.\right. \\
& C_{2}=\left\{(r \cos \theta, r \sin \theta) \mid(r, \theta) \in\left[0,+\infty\left[\times\left[\frac{\pi}{2}-\theta_{1}, \frac{\pi}{2}+\theta_{1}\right]\right\} ;\right.\right. \\
& C_{3}=\left\{(r \cos \theta, r \sin \theta) \mid(r, \theta) \in\left[0,+\infty\left[\times\left[-\frac{\pi}{2}-\theta_{1},-\frac{\pi}{2}+\theta_{1}\right]\right\} ;\right.\right. \\
& C_{4}=\left\{(r \cos \theta, r \sin \theta) \mid(r, \theta) \in\left[0,+\infty\left[\times\left(\left[-\pi,-\pi+\theta_{1}\right] \cup\left[\pi-\theta_{1}, \pi\right]\right)\right\} .\right.\right.
\end{aligned}
$$

This example is illustrated by the following figure.

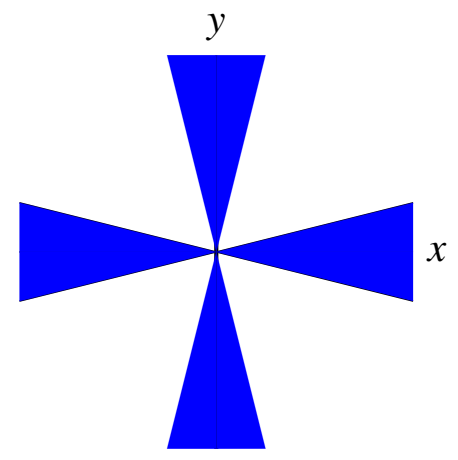


FIGURE 1. Example of the weak normal cone in $\mathbb{R}^{2}$

Example 2.5. The geometry of $w-\mathrm{N}_{D}^{\alpha}$ is variable depending on the subset $D$. To clarify this, again in $X=\mathbb{R}^{2}$ but with $D=\left\{(x, y) \in \mathbb{R}^{2} \mid y=x\right\}$ and $\alpha=\frac{1}{2}$, a similar use of polar coordinates immediately shows that $w-\mathrm{N}_{D}^{\frac{1}{2}}(0,0)$ is nothing else but the line of equation $y=-x$.

This new definition of the weak approximate normal cone accordingly generates a new concept of weak approximate quasi-variational inequalities which we introduce in the next definition.

Definition 2.1. Let $\alpha>0$. The weak quasi-variational inequality problem associated with $\mathrm{QVI}(T, \mathbb{K})$ is defined as follows: Find $\bar{x} \in \mathscr{K}$ such that $d(\bar{x}, \mathbb{K}(\bar{x})) \leq \alpha$ and $x^{*} \in T(\bar{x})$ such that

$$
(w, \alpha)-\mathrm{QVI}(T, \mathbb{K}):-x^{*} \in w-\mathrm{N}_{\mathbb{K}(\bar{x})}^{\alpha}(\bar{x}) .
$$

where the notation $(w, \alpha)$ refers to weak solutions because they are only approximate fixed points of $\mathbb{K}$.

Thus, for any $\alpha>0$, a point $\bar{x} \in \mathscr{K}$ is a weak $\alpha$-approximate solution to $\mathrm{QVI}(T, \mathbb{K})$ if and only if $\bar{x}$ is a solution to $(w, \alpha)-\mathrm{QVI}(T, \mathbb{K})$, i.e., $\bar{x} \in \mathscr{K}$ with $d(\bar{x}, \mathbb{K}(\bar{x})) \leq \alpha$ and there exists $x^{*} \in T(\bar{x})$ such that

$$
\left\langle x^{*}, y-\bar{x}\right\rangle \geq-\alpha\left\|x^{*}\right\|, \forall y \in \mathbb{K}(\bar{x}) .
$$

Example 2.6. In the last section of this paper, we illustrate problem $(w, \alpha)-\mathrm{QVI}(T, \mathbb{K})$ by a general model of weak Social Nash Games. Let us now give three particular cases:

(1) If $T=0$, i.e., $T(x)=0$ for all $x \in X$, then clearly $(w, \alpha)$-QVI $(0, \mathbb{K})$ coincides with the classic problem of $\alpha$-approximate fixed points of the set-valued map $\mathbb{K}$.

(2) If $T$ is a single-valued operator and $\mathbb{K}$ is a constant map, i.e., for some subset $K \subset \mathscr{K}$, $\mathbb{K}(x)=K$, then $(w, \alpha)-\mathrm{QVI}(T, \mathbb{K})$ turns out to be a weak approximation of the standard variational inequality: Find $\bar{x} \in K$ such that

$$
\mathrm{VI}(T, K) \quad\langle T(\bar{x}), y-\bar{x}\rangle \geq 0, \forall y \in K .
$$

(3) In the last example, if we take $T=\nabla f$, the gradient of a given continuously differentiable function $f$, then the corresponding variational inequality $\operatorname{VI}(\nabla f, K)$ expresses optimality conditions of the problem of minimizing $f$ over the set of constraints $K$. In this case, a minimum of $f$ can be obtained as a limit of a sequence of weak minima $\left(x_{\alpha}\right)_{\alpha}$ in the following sense: for every $\alpha>0$, there exists $x_{\alpha} \in K$ such that

$$
\alpha-\operatorname{VI}(\nabla f, K) \quad\left\langle\nabla f\left(x_{\alpha}\right), y-x_{\alpha}\right\rangle \geq-\alpha\left\|\nabla f\left(x_{\alpha}\right)\right\|, \forall y \in K
$$

Our approach constructs weak approximate solutions to (QVI) by regularizing the closed convex hull of the solution map $S$ associated with QVI $(T, \mathbb{K})$; see Theorem 4.1 below.

\section{FURTHER SET-VALUED ANALYSIS ELEMENTS}

Let $X$ and $Y$ be two normed vector spaces. Their norms are denoted by $\|$.$\| . For any nonempty$ subset $A$ of $X$ and any point $x \in X, d(x, A)=\inf \{\|x-y\|: y \in A\}$ will stand for the distance 
from $x$ to $A$ where $d(x, \emptyset)=\infty$. If $B$ is another nonempty subset of $X, e(A, B)$ denotes the excess of $A$ on $B$ given by

$$
e(A, B)=\sup \{d(a, B): a \in A\} .
$$

A set-valued map $\Gamma: X \rightrightarrows Y$ is said to be Hausdorff upper (resp. lower) continuous, $H$-u.c (resp. $H$-l.c) in short, at a point $x_{0} \in X$ if and only if

$$
\left.\lim _{x \rightarrow x_{0}} e\left(\Gamma(x), \Gamma\left(x_{0}\right)\right)=0 \text { (resp. } \lim _{x \rightarrow x_{0}} e\left(\Gamma\left(x_{0}\right), \Gamma(x)\right)=0\right) \text {. }
$$

If $\Gamma$ is $H$-u.c (resp. $H$-l.c) at any point $x \in X$, then $\Gamma$ is said to be $H$-u.c (resp. $H$-1.c). Obviously, every Lipschitz set-valued map is $H$-u.c and $H$-l.c. If a map is $H$-u.c and $H$-l.c, then it is said to be Hausdorff continuous. The Hausdorff continuity is also called in the literature Hausdorff semicontinuity.

We denote by $B_{r}\left(\operatorname{resp} \bar{B}_{r}\right)$ the open (resp. closed) ball centered at 0 with radius $r$ in any of the spaces $X, Y$. Let us consider a set-valued map $S: X \rightrightarrows Y$. When $S(x) \neq \emptyset$, we say that $x$ is in the domain of $S$, i.e., $x \in \operatorname{Dom}(S)$. The lower limit of $S$ at a point $\bar{x} \in \operatorname{Dom}(S)$ is defined as follows:

$$
\liminf _{x \rightarrow \bar{x}} S(x)=\left\{y \in Y \mid \forall x_{n} \rightarrow \bar{x}, \exists y_{n} \rightarrow y, y_{n} \in S\left(x_{n}\right), \forall n \geq n_{0} \text { for some } n_{0}\right\} .
$$

Note that, if $\bar{x} \notin \operatorname{Dom}(S)$, then $\liminf _{x \rightarrow \bar{x}} S(\bar{x})=\emptyset$.

Note that a point $y \in Y$ belongs to $\liminf _{x \rightarrow \bar{x}} S(x)$ if and only if for all sequence $\left(x_{n}\right)$ converging to $\bar{x}$,

$$
\lim _{n \rightarrow+\infty} d\left(y, S\left(x_{n}\right)\right)=\limsup _{n \rightarrow+\infty} d\left(y, S\left(x_{n}\right)\right)=0 .
$$

A set-valued map $S: X \rightrightarrows Y$ is said to be lower semicontinuous at $\bar{x} \in \operatorname{Dom}(S)$ if

$$
S(\bar{x}) \subseteq \liminf _{x \rightarrow \bar{x}} S(x)
$$

If $\bar{x} \notin \operatorname{Dom}(S)$, then one considers that $S$ is automatically l.s.c. at $\bar{x}$.

The space $Y$ will be partially ordered by a closed, convex (not necessarily pointed) cone $C$ with nonempty interior. Then, for a set-valued mapping $S: X \rightrightarrows Y$ and $\varepsilon>0$, we consider $S^{\varepsilon}: X \rightrightarrows Y$ defined for each $x \in X$ by

$$
S^{\varepsilon}(x)=S(x)-C_{\varepsilon},
$$

where $C_{\varepsilon}=C \cap \bar{B}_{\varepsilon}$. We use the convention $\emptyset-C_{\varepsilon}=\emptyset$, so that

$$
\operatorname{Dom}(S)=\operatorname{Dom}\left(S^{\varepsilon}\right) \text {. }
$$

Let us introduce $L_{S}^{\varepsilon}: X \rightrightarrows Y$ as a set-valued map defined for each $\bar{x} \in X$ by

$$
L_{S}^{\varepsilon}(\bar{x})=\liminf _{x \rightarrow \bar{x}} S^{\varepsilon}(x) .
$$

If $\bar{x} \notin \operatorname{Dom}(S)$, then $L_{S}^{\varepsilon}(\bar{x})=\emptyset$. We recall the result of [1], where it was proved that $L_{S}^{\varepsilon}$ satisfies a property weaker than lower semicontinuity. Indeed, for every $\varepsilon \geq 0, \eta>0$, one has

$$
L_{S}^{\varepsilon}(\bar{x}) \subseteq \liminf _{x \rightarrow \bar{x}} L_{S}^{\varepsilon+\eta}(x), \forall \bar{x} \in X .
$$

Let $\alpha$ be a positive number. We introduce the set-valued map $R_{S}^{\alpha}(\bar{x}): X \rightrightarrows Y$, given in [1] by

$$
R_{S}^{\alpha}(\bar{x}):=\operatorname{cl}\left(\underset{\tau \in[0, \alpha)}{\cup} \liminf _{x \rightarrow \bar{x}} L_{S}^{\tau}(x)\right) .
$$


It was shown in [1] that $R_{S}^{\alpha}$ is lower semicontinuous, and it is called lower semicontinuous regularization or $(C, \alpha)$-regularization of $S$. Notice that if $S$ is convex-valued, then, for any positive real number $\alpha$, the associated regularization $R_{S}^{\alpha}$ at $\bar{x}$ is convex (for more details, see the proof of [1, Theorem 5.3]). Given a set-valued map $S: X \rightrightarrows Y$, we consider its convex hull map $\operatorname{conv} S: X \rightrightarrows Y$ defined by $\operatorname{conv} S(x)=\operatorname{conv}(S(x))$, and its closure map $\bar{S}: X \rightrightarrows Y$ defined by $\bar{S}(x)=\overline{S(x)}$. It is clear that $\overline{c o n v S}$ is closed and convex-valued.

We equally need the following proposition, which was also proved in [1].

Proposition 3.1. Let $F: X \rightrightarrows Y$ be a set-valued map and $\bar{x} \in \operatorname{Dom}(F)$. If one of the following conditions is satisfied:

1. $F(\bar{x})$ is compact;

2. $Y$ is reflexive and $F(\bar{x})$ is weakly closed;

3. $F(\bar{x})$ is convex, closed, locally compact,

then, for every $\alpha>0$,

$$
\liminf _{x \rightarrow \bar{x}} F(x) \subset R_{F}^{\alpha}(\bar{x}) \subseteq F(\bar{x})-C_{\alpha} .
$$

From Proposition 3.1, we see that $R_{F}^{\alpha}(\bar{x})$ is nonempty whenever $\liminf _{x \rightarrow \bar{x}} F(x)$ is so. In the following Lemma, we show that the nonemptiness of the lower limit of closed valued maps is always satisfied whenever its image is contained in a compact subset of the range space.

Lemma 3.1. Let $X$ and $Y$ be normed vector spaces, and let $F: X \rightrightarrows Y$ be a closed-valued multimap. Assume that there exists a compact $K$ of $Y$ such that $F(x) \subset K$ for all $x \in \operatorname{Dom}(F)$. Then, for all $\bar{x} \in \operatorname{Dom}(F), R_{F}^{\alpha}(\bar{x})$ is nonempty for every $\alpha>0$.

Proof. Fix some $\alpha>0$. Let $\bar{x} \in \operatorname{Dom}(F)$. Take a sequence $\left(x_{n}\right)$ converging to $\bar{x}$ and define, for all $n, y_{n}:=\Pi_{F\left(x_{n}\right)}\left(x_{n}\right)$, where, for a closed subset $C$ of $Y$, and a given $u \in Y, \Pi_{C}(u)$ denotes the metric projection onto the closed set $C$ given by

$$
\Pi_{C}(u):=\{x \in C:\|x-u\|=d(u, C)\} .
$$

Obviously, for all $n, y_{n} \in F\left(x_{n}\right) \subset K$. Then, $\left(y_{n}\right)_{n}$ admits a subsequence, denoted again by $\left(y_{n}\right)$, converging to some $y \in K$. Since, for all $n, y_{n} \in F\left(x_{n}\right)$, it results that

$$
d\left(y, F\left(x_{n}\right)\right) \leq\left\|y-y_{n}\right\|_{Y}, \forall n
$$

where $\|\cdot\|_{Y}$ is the norm of $Y$. This implies that $\lim \sup d\left(y, F\left(x_{n}\right)\right)=0$, which together with (3.1) gives

This achieves the proof.

$$
y \in \liminf _{x \rightarrow \bar{x}} F(x) \subset R_{F}^{\alpha}(\bar{x}) \neq \emptyset .
$$

We end this section by three fundamental classic ingredients.

Theorem 3.1. (Michael) Let $X$ be a metric space, $Y$ be a Banach space and $F: X \rightrightarrows Y$ be a l.s.c set-valued mapping with nonempty, closed, convex values. Then $F$ admits a continuous selection.

Theorem 3.2. [6, Theorem 6.4.5] Let $\mathscr{K}$ be a nonempty compact convex subset of a locally convex space $X$, and let $S$ be a set-valued map from $\mathscr{K}$ to $\mathscr{K}$ such that

i) for each $x \in \mathscr{K}, S^{-1}(\{x\})=\{c \in \mathscr{K}: x \in S(c)\}$ is open in $\mathscr{K}$; 
ii) $S(c)$ is nonempty and convex for each $c \in \mathscr{K}$.

Then $S$ has a fixed point.

Theorem 3.3. (Sion's minimax Theorem) Let X be a compact convex subset of a linear topological space and $Y$ a convex subset of a linear topological space. Let $f$ be a real-valued function on $X \times Y$ such that

i) $f(x, \cdot)$ is upper semicontinuous and quasi-concave on $Y, \forall x \in X$;

ii) $f(\cdot, y)$ is lower semicontinuous and quasi-convex on $X, \forall y \in Y$.

Then,

$$
\min _{x \in X} \sup _{y \in Y} f(x, y)=\sup _{y \in Y} \min _{x \in X} f(x, y) .
$$

\section{MAin RESUlTS}

4.1. Weak approximate solutions to (QVI) problems. To establish our first result, we need the following Lemma.

Lemma 4.1. Let $\mathscr{K}$ be a nonempty, compact and convex subset of a locally convex space $X$, and let $W$ be an open convex neighborhood of the origin. Suppose that $f: \mathscr{K} \rightarrow X$ is continuous and $f(\mathscr{K}) \subset \mathscr{K}-W$. Then there exists $c \in \mathscr{K}$ satisfying $f(c) \in c-W$.

Proof. Consider the set-valued map $S$ mapping from $\mathscr{K}$ to $\mathscr{K}$ defined by $S(x)=(f(x)+W) \cap$ $\mathscr{K}, x \in \mathscr{K}$. Since $f(x) \in \mathscr{K}-W$, we have that $S(x)$ is nonempty for each $x \in \mathscr{K}$. Moreover, $S(x)$ is convex as an intersection of two convex sets. By the continuity of $f, S^{-1}(\{x\})$ is open in $\mathscr{K}$ for each $x \in \mathscr{K}$. Then, by Theorem 3.2, $S$ has a fixed point $c \in \mathscr{K}$, which implies that $f(c) \in c-W$. This completes the proof.

Theorem 4.1. Let $X$ be a normed space, $\mathscr{K}$ be a convex compact and nonempty subset of $X$, and let $T: \mathscr{K} \rightrightarrows X^{*}$ be a set-valued map satisfying the following conditions:

(i) $T(x)$ is nonempty, convex and compact, for all $x \in \mathscr{K}$;

(ii) $\mathbb{K}(x)$ is convex and closed, for all $x \in \mathscr{K}$.

Then $\mathrm{QVI}(T, \mathbb{K})$ admits a weak $\alpha$-approximate solution for every $\alpha>0$.

Proof. Let $\alpha>0$. Fix $\mu$ in $] 0, \frac{\alpha}{2}[$. Since $\mathbb{K}(x)$ is convex and closed, for all $x \in \mathscr{K}$, we can write

$$
\overline{\operatorname{convS}}(x)=\overline{\operatorname{convS}(x)} \subset \mathbb{K}(x) \subset \mathscr{K} .
$$

This implies that, for each $x \in \mathscr{K}, \overline{\operatorname{convS}}(x)$ is a convex and compact subset of $\mathscr{K}$. Then, by Lemma 3.1, $\frac{\mu}{\operatorname{convS}}(x) \neq \emptyset$ for all $x \in \mathscr{K}$. Moreover, by Proposition 3.1, we obtain

$$
\begin{aligned}
R_{\overline{c o n v} S}^{\mu}(x) & \subset \overline{\operatorname{conv} S}(x)-C_{\mu} \\
& \subset \mathbb{K}(x)-C_{\mu} \\
& \subset \mathscr{K}-C_{\frac{\alpha}{2}} .
\end{aligned}
$$

Given that the set-valued map $R_{\frac{1}{\operatorname{convS}}}^{\mu}: \mathscr{K} \rightrightarrows \mathscr{K}-C_{\frac{\alpha}{2}}$ is lower semicontinuous with nonempty, convex and closed values, we find from Michael's selection Theorem that there exists a continuous function $f_{\mu}$ :

$$
f_{\mu}: \mathscr{K} \rightarrow \mathscr{K}-C_{\frac{\alpha}{2}} \subset \mathscr{K}-B \frac{\alpha}{2}
$$


such that

$$
f_{\mu}(x) \in R_{\overline{\operatorname{conv} S}}^{\mu}(x), \forall x \in \mathscr{K} .
$$

Hence, in view of Lemma 4.1, there exists $x_{\alpha} \in \mathscr{K}$ such that

$$
f_{\mu}\left(x_{\alpha}\right)=x_{\alpha}-b_{\alpha}
$$

i.e.,

with $b_{\alpha} \in B \frac{\alpha}{2}$.

$$
x_{\alpha}=f_{\mu}\left(x_{\alpha}\right)+b_{\alpha}
$$

Now, from the inclusion

we infer

$$
R_{\overline{\operatorname{convS}}}^{\mu}(x) \subseteq \overline{\operatorname{convS}}(x)-C_{\frac{\alpha}{2}}
$$

$$
f_{\mu}\left(x_{\alpha}\right) \in \overline{\operatorname{conv} S\left(x_{\alpha}\right)}-C_{\frac{\alpha}{2}} .
$$

This implies in turn the existence of some $a_{\alpha} \in \overline{\operatorname{conv} S\left(x_{\alpha}\right)}$ and $d_{\alpha} \in C_{\frac{\alpha}{2}}$ such that

$$
x_{\alpha}=a_{\alpha}-\left(d_{\alpha}-b_{\alpha}\right)=a_{\alpha}-c_{\alpha},
$$

where $c_{\alpha} \in B_{\alpha}$. Clearly, there exists a sequence $\left(a_{n}\right)_{n}$ converging to $a_{\alpha}$ such that

$$
a_{n} \in \operatorname{conv} S\left(x_{\alpha}\right), \forall n \text {. }
$$

For each $n$, we find from the definition of $\operatorname{conv} S\left(x_{\alpha}\right)$ that there exist $\lambda_{1}, \ldots, \lambda_{p} \in[0,1]$ and $a_{n 1}, \ldots, a_{n p} \in S\left(x_{\alpha}\right)$ such that

$$
a_{n}=\sum_{i=1}^{p} \lambda_{i} a_{n i} \text { and } \sum_{i=1}^{p} \lambda_{i}=1 .
$$

Thus, from the definition of $S\left(x_{\alpha}\right)$, for each $i \in\{1, \cdots, p\}$, we have that there exists $a_{n i}^{*} \in T\left(x_{\alpha}\right)$ such that

$$
\left\langle a_{n i}^{*}, y-a_{n i}\right\rangle \geq 0, \forall y \in \mathbb{K}\left(x_{\alpha}\right) .
$$

Let us introduce the following notation

$$
x_{n}=a_{n}-c_{\alpha} \text { and } x_{n}^{*}=\sum_{i=1}^{p} \lambda_{i} a_{n i}^{*} .
$$

With these elements, we claim that

$$
\left\langle x_{n}^{*}, y-x_{n}\right\rangle \geq-\alpha\left\|x_{n}^{*}\right\|, \forall y \in \mathbb{K}\left(x_{\alpha}\right) .
$$

Let us prove this claim. For simplicity, we denote $a_{n i}$ by $a_{i}$ and $a_{n i}^{*}$ by $a_{i}^{*}$. Let $i \in\{1, \ldots, p\}$. Then the inequality (4.3) becomes

$$
\left\langle a_{i}^{*}, y-a_{i}\right\rangle \geq 0, \forall y \in \mathbb{K}\left(x_{\alpha}\right)
$$

Hence,

$$
\lambda_{i}\left\langle a_{i}^{*}, y-a_{n}\right\rangle+\lambda_{i}\left\langle a_{i}^{*}, a_{n}-a_{i}\right\rangle \geq 0, \forall y \in \mathbb{K}\left(x_{\alpha}\right) .
$$

If we pass to the sum over $i \in\{1, \cdots, p\}$ in the previous inequality, we obtain that, for all $y \in \mathbb{K}\left(x_{\alpha}\right)$,

$$
\sum_{i=1}^{p} \lambda_{i}\left\langle a_{i}^{*}, y-a_{n}\right\rangle+\sum_{i=1}^{p} \lambda_{i}\left\langle a_{i}^{*}, a_{n}-a_{i}\right\rangle \geq 0 .
$$


Thus, we get

$$
\left\langle\sum_{i=1}^{p} \lambda_{i} a_{i}^{*}, y-a_{n}\right\rangle+\sum_{i=1}^{p} \lambda_{i}\left\langle a_{i}^{*}, a_{n}-a_{i}\right\rangle \geq 0, \quad \forall y \in \mathbb{K}\left(x_{\alpha}\right) .
$$

Therefore

$$
\left\langle x_{n}^{*}, y-a_{n}\right\rangle+\sum_{i=1}^{p} \lambda_{i}\left\langle a_{i}^{*}, a_{n}-a_{i}\right\rangle \geq 0, \forall y \in \mathbb{K}\left(x_{\alpha}\right) .
$$

Next, we justify that there exists $x^{*} \in T\left(x_{\alpha}\right)$ such that

$$
\left\langle x^{*}, a_{n}-a_{i}\right\rangle \geq\left\langle a_{i}^{*}, a_{n}-a_{i}\right\rangle, \quad \forall i \in\{1, \cdots, p\} .
$$

For each $i \in\{1, \cdots, p\}$, we consider the set $A_{i}$ defined by

$$
A_{i}=\left\{y^{*} \in T\left(x_{\alpha}\right) \mid\left\langle y^{*}-a_{i}^{*}, a_{n}-a_{i}\right\rangle \geq 0\right\} .
$$

Clearly, for every $i \in\{1, \cdots, p\}, A_{i}$ is nonempty (as $a_{i}^{*} \in A_{i}$ ), it is also trivially closed. Let us check that $\underset{i=1}{p} A_{i} \neq \emptyset$. For a contradiction, we assume that $\bigcap_{i=1}^{p} A_{i}=\emptyset$. Then

$$
T\left(x_{\alpha}\right)=\bigcup_{i=1}^{p} A_{i}^{c}
$$

where $A_{i}^{c}$ denotes the complement of $A_{i}$ in $T\left(x_{\alpha}\right)$. This implies that $T\left(x_{\alpha}\right)$ is an open set as a finite union of open subsets, which is in conflict with the compactness condition supposed on $T$. Consequently, any element $x^{*} \in \bigcap_{i=1}^{p} A_{i}$ satisfies the required inequality in (4.10).

In the following, (4.9) and (4.10) give us

$$
\left\langle x_{n}^{*}, y-a_{n}\right\rangle+\sum_{i=1}^{p} \lambda_{i}\left\langle x^{*}, a_{n}-a_{i}\right\rangle \geq 0, \forall y \in \mathbb{K}\left(x_{\alpha}\right) .
$$

Then

$$
\left\langle x_{n}^{*}, y-a_{n}\right\rangle+\left\langle x^{*}, \sum_{i=1}^{p} \lambda_{i}\left(a_{n}-a_{i}\right)\right\rangle \geq 0, \forall y \in \mathbb{K}\left(x_{\alpha}\right) .
$$

Thus, by involving (4.2) in the last inequality, we see that

$$
\left\langle x_{n}^{*}, y-a_{n}\right\rangle \geq 0, \quad \forall y \in \mathbb{K}\left(x_{\alpha}\right) .
$$

But $x_{n}=a_{n}-c_{\alpha}$. It follows that

$$
\left\langle x_{n}^{*}, y-\left(x_{n}+c_{\alpha}\right)\right\rangle \geq 0 .
$$

This means that

$$
\left\langle x_{n}^{*}, y-x_{n}\right\rangle \geq\left\langle x_{n}^{*}, c_{\alpha}\right\rangle \geq-\alpha\left\|x_{n}^{*}\right\| .
$$

The claim (4.5) is then proved. At this stage of the proof, taking into account the facts that $T\left(x_{\alpha}\right)$ is convex and $a_{i}^{*} \in T\left(x_{\alpha}\right)$ for each $i \in\{1, \ldots, p\}, x_{n}^{*} \in T\left(x_{\alpha}\right)$, for all $n$. Thus, the compactness of $T\left(x_{\alpha}\right)$ ensures that $\left(x_{n}^{*}\right)_{n}$ admits a subsequence $\left(x_{n_{k}}^{*}\right)_{k}$ that converges to some $x_{\alpha}^{*} \in T\left(x_{\alpha}\right)$. Of course, for all $k$, we have

$$
\left\langle x_{n_{k}}^{*}, y-x_{n_{k}}\right\rangle \geq-\alpha\left\|x_{n_{k}}^{*}\right\|, \quad \forall y \in \mathbb{K}\left(x_{\alpha}\right) .
$$


Moreover, $\left(x_{n}\right)_{n}$ converges to $x_{\alpha}$ (since $\left(a_{n}\right)_{n}$ converges to $a_{\alpha}$ ). Accordingly, with the limit over $k \rightarrow+\infty$ in (4.11), we arrive at

$$
\left\langle x_{\alpha}^{*}, y-x_{\alpha}\right\rangle \geq-\alpha\left\|x_{\alpha}^{*}\right\|, \forall y \in \mathbb{K}\left(x_{\alpha}\right) .
$$

Finally, as $a_{\alpha} \in \overline{\operatorname{conv} S}\left(x_{\alpha}\right) \subset \mathbb{K}\left(x_{\alpha}\right)$ and $x_{\alpha}=a_{\alpha}-c_{\alpha}$, it follows that

$$
d\left(x_{\alpha}, \mathbb{K}\left(x_{\alpha}\right)\right) \leq\left\|a_{\alpha}-x_{\alpha}\right\|=\left\|c_{\alpha}\right\| \leq \alpha .
$$

Therefore, with (4.12), we conclude that $x_{\alpha}$ is an $\alpha$-weak solution to $\mathrm{QVI}(\mathbb{K}, T)$. This completes the proof.

Remark 4.1. Let us underline that this Theorem still holds if we make a slight change in the proof of the auxiliary step (4.10) as follows. For some $\eta>0$,

$$
\left\langle x^{*}, a_{n}-a_{i}\right\rangle \geq\left\langle a_{i}^{*}, a_{n}-a_{i}\right\rangle+\eta, \forall i \in\{1, \cdots, p\} .
$$

This ensures that the solution $x_{\alpha}$ may be considered nontrivial in the sense that the corresponding dual element $x_{\alpha}^{*} \neq 0$. Observe that the cone $C$ is arbitrary chosen (with nonempty interior). Then, in view of (4.1), provided that $C$ has no intersection with $\mathscr{K}$ (or with the set of fixed points of $\mathbb{K}$, in particular, this holds if $C \cap \mathbb{K}(x)=\emptyset$, for every $x \in \mathscr{K}$ ), a fortiori $x_{\alpha} \neq 0$.

Remark 4.2. In view of Remark 4.1, Theorem 4.1 can be envisaged for applications to quasiconvex programming, where $T$ plays the role of the normal operator $N_{f}^{a}$ defined by the normal cone to an adjusted sublevel set of a function $f$ to be optimized (see [4, Section 5]).

4.2. Exact solutions to (QVI). In this subsection, we provide exact solutions to our quasivariational inequality problem under a convergence argument of the weak approximate solutions established in Theorem 4.1. To this end, we need a qualitative stability result of approximate fixed points of the map $\mathbb{K}$. Let us state and prove this property for any upper semicontinuous set-valued in the general setting of metric spaces.

Lemma 4.2. Let $(X, \rho)$ be a metric space, and let $F: X \rightrightarrows X$ be a set-valued mapping with closed values. Assume that, for every $\varepsilon>0$, there exists $x_{\varepsilon} \in X$ such that $d\left(x_{\varepsilon}, F\left(x_{\varepsilon}\right)\right) \leq \varepsilon$, and suppose moreover that $F$ is Hausdorff upper continuous on $\mathscr{K}$, i.e., for all $\bar{x} \in \mathscr{K}$,

$$
\lim _{x \rightarrow \bar{x}} e(F(x), F(\bar{x}))=0 .
$$

Then whenever $\left(x_{\varepsilon}\right)$ converges to some point $\bar{x} \in \mathscr{K}$, as $\varepsilon$ goes to $0, \bar{x}$ is a fixed point of $F$, i.e., $\bar{x} \in F(\bar{x})$.

Proof. Let $\left(x_{\varepsilon}\right)_{\varepsilon}$ be a sequence of approximate fixed points of $F$. For an arbitrary $\varepsilon>0$, take any element $y_{\varepsilon} \in F\left(x_{\varepsilon}\right)$. Visibly,

$$
\begin{aligned}
d(\bar{x}, F(\bar{x})) & \leq \rho\left(\bar{x}, x_{\varepsilon}\right)+\rho\left(x_{\varepsilon}, y_{\varepsilon}\right)+d\left(y_{\varepsilon}, F(\bar{x})\right) \\
& \leq \rho\left(\bar{x}, x_{\varepsilon}\right)+\rho\left(x_{\varepsilon}, y_{\varepsilon}\right)+e\left(F\left(x_{\varepsilon}\right), F(\bar{x})\right) .
\end{aligned}
$$

By passing to infimum over $y_{\varepsilon}$ in $F\left(x_{\varepsilon}\right)$ in the previous inequality, we obtain

$$
\begin{aligned}
d(\bar{x}, F(\bar{x})) & \leq \rho\left(\bar{x}, x_{\varepsilon}\right)+d\left(x_{\varepsilon}, F\left(x_{\varepsilon}\right)\right)+e\left(F\left(x_{\varepsilon}\right), F(\bar{x})\right) \\
& \leq \rho\left(\bar{x}, x_{\varepsilon}\right)+\varepsilon+e\left(F\left(x_{\varepsilon}\right), F(\bar{x})\right) .
\end{aligned}
$$


Therefore, by involving the convergence of $\left(x_{\varepsilon}\right)$ to $\bar{x}$ when $\varepsilon$ goes to 0 and the Hausdorff upper continuity of $F$ at $\bar{x}$, we deduce that

$$
d(\bar{x}, F(\bar{x}))=0 .
$$

Since $F$ is closed-valued, $F(\bar{x})$ is closed, and hence $\bar{x} \in F(\bar{x})$. This shows that $\bar{x}$ is a fixed point of $F$. This completes the proof.

We are now ready to present our result on the existence of exact solutions to QVI $(T, \mathbb{K})$.

Theorem 4.2. Suppose that the assumptions of Theorem 4.1 are fulfilled and moreover the following conditions are verified:

a) the map $\mathbb{K}$ is Hausdorff continuous;

b) $(\mathbb{H})$ : for all $x, y \in \mathscr{K}$, for all $x_{\alpha} \longrightarrow x$, for all $y_{\alpha} \longrightarrow y$,

$$
\liminf _{\alpha \rightarrow 0} \sup _{x_{\alpha}^{\star} \in T\left(x_{\alpha}\right)}\left\langle x_{\alpha}^{\star}, y_{\alpha}-x_{\alpha}\right\rangle \geq 0 \Longrightarrow \sup _{x^{\star} \in T(x)}\left\langle x^{\star}, y-x\right\rangle \geq 0 .
$$

Then $\mathrm{QVI}(T, \mathbb{K})$ admits an exact solution.

Proof. From Theorem 4.1, there exists a net $\left(x_{\alpha}\right)_{\alpha}$ of weak $\alpha$-approximate solutions to QVI $(T, \mathbb{K})$. Thanks to the compactness of $\mathscr{K}$, this net admits a subnet, also denoted by $\left(x_{\alpha}\right)$, converging to some point $\bar{x} \in \mathscr{K}$. Obviously, (by the definition of weak solutions), for all $\alpha>0$,

$$
d\left(x_{\alpha}, \mathbb{K}\left(x_{\alpha}\right)\right) \leq \alpha .
$$

Therefore, from the assumption a) and Lemma 4.2, it results that $\bar{x} \in \mathbb{K}(\bar{x})$.

Moreover, as $\mathbb{K}$ is $H$-l.c (Hausdorff lower continuous) and compact-valued, $\mathbb{K}$ is lower semicontinuous (see [7, p. 59]). Thus, for a fixed element $y \in \mathbb{K}(\bar{x})$, there exists a net $\left(y_{\alpha}\right)_{\alpha}$, such that $y_{\alpha} \in \mathbb{K}\left(x_{\alpha}\right)$, converging to $y$. By the definition of $\left(x_{\alpha}\right)_{\alpha}$, for all $\alpha>0$, there exists $x_{\alpha}^{\star} \in T\left(x_{\alpha}\right)$ such that

$$
\left\langle x_{\alpha}^{\star}, y_{\alpha}-x_{\alpha}\right\rangle \geq-\alpha\left\|x_{\alpha}^{\star}\right\|
$$

Therefore, the condition $(\mathbb{H})$ leads to

$$
\sup _{x^{*} \in T(\bar{x})}\left\langle x^{*}, y-\bar{x}\right\rangle \geq 0 \text {. }
$$

This gives us

$$
\inf _{y \in \mathbb{K}(\bar{x})} \sup _{x^{*} \in T(\bar{x})}\left\langle x^{*}, y-\bar{x}\right\rangle \geq 0 .
$$

On the other hand, by applying the Sion's minimax theorem, we have

$$
\inf _{y \in \mathbb{K}(\bar{x})} \sup _{x^{*} \in T(\bar{x})}\left\langle x^{*}, y-x\right\rangle=\sup _{x^{*} \in T(\bar{x})} \inf _{y \in \mathbb{K}(\bar{x})}\left\langle x^{*}, y-x\right\rangle .
$$

But the function $x^{*} \mapsto \inf _{y \in \mathbb{K}(\bar{x})}\left\langle x^{*}, y-\bar{x}\right\rangle$ is upper semicontinuous and $T(\bar{x})$ is compact. It follows that there exists $\overline{x^{*}} \in T(\bar{x})$ such that

$$
\left\langle\overline{x^{*}}, y-\bar{x}\right\rangle \geq 0, \forall y \in \mathbb{K}(\bar{x}),
$$

which means that $\bar{x}$ is a solution of $\mathrm{QVI}(T, \mathbb{K})$. This achieves the proof.

Remark 4.3. (1) In view of the compactness assumption on $\mathbb{K}$, the semicontinuity assumption of this map coincides with its continuity in the sense of Hausdorff (see [7, Chapter 2]). 
(2) The regularity property $(\mathbb{H})$ is suitable for the convergence of both weak and strong solutions, it was introduced in [2] by inspiration from the dual lower semicontinuity of $T$ on $\mathscr{K}$ in the sense of [8, Definition 3.1] (see also [4]). For a detailed discussion on this condition together with general and explicit examples, we refer to [2].

(3) In the convergence result of Theorem 4.2 above, the condition $(\mathbb{H})$ can also be replaced with a slight change in the condition ii) of [9, Theorem 1] (see also [10]): For each $y \in \mathscr{K}-\mathscr{K},\left\{x \in \mathscr{K}: \inf _{x^{*} \in T(x)}\left\langle x^{*}, y\right\rangle \leq 0\right\}$ is closed.

\section{WEAK $\alpha$-APPROXIMATE SOLUTION FOR THE SOCIAL NASH EQUILIBRIUM PROBLEM}

Let $Y_{i}$ be a Banach space, $i=1,2 . ., n$. Let us consider the well-known model of $n$-players in noncooperative games with coupled constraints; see, for example, $[11,12,13]$. A player $i$ chooses an action $x_{i}$ in a set $X_{i} \subset Y_{i}$, called the strategy set, and seeks to the optimize individually and noncooperatively his payoff function, say $J_{i}: X=X_{1} \times \ldots \times X_{n} \longrightarrow \mathbb{R}$, but cannot escape the effects of his rivals's choices. The $n$-tuple of actions $\left(x_{1}, \ldots, x_{n}\right)$, denoted by $x$, is an element of $X=X_{1} \times \ldots \times X_{n}$. We adopt the notation $x_{-i}$ given by

$$
x_{-i}=\left(x_{1}, x_{2}, \ldots, x_{i-1}, x_{i+1}, . ., x_{n}\right),
$$

which is standard in game theory. The constraints of a player $i$ depends, as mentioned, on the strategies of the other players and given by

$$
K_{i}\left(x_{-i}\right)=\left\{y_{i} \in X_{i} \mid f_{j}^{i}\left(y_{i}, x_{-i}\right) \leq 0, \text { for all } j=1,2, \ldots, m_{i}\right\},
$$

where $f_{j}^{i}\left(., x_{-i}\right): X_{i} \rightarrow \mathbb{R}$ is a proper, closed and convex function for all $j=1,2, \ldots, m_{i}$ and all $i=1,2, . ., n$.

Definition 5.1. We say that a point $\bar{x} \in X$ is a social Nash equilibrium of the game $\left(X_{i}, J_{i}, K_{i}\right)_{1 \leq i \leq n}$ if for all $i=1,2 . ., n$,

$$
J_{i}(\bar{x}) \leq J_{i}\left(x_{i}, \bar{x}_{-i}\right) \text { for all } x_{i} \in K_{i}\left(\bar{x}_{-i}\right)
$$

This problem of Nash equilibria is equivalent to the following system of inequalities (see, for example, $[14,15,16,17])$ : Find $\bar{x} \in X$ with $\bar{x} \in K_{1}\left(\bar{x}_{-1}\right) \times \ldots \times K_{n}\left(\bar{x}_{-n}\right)$ such that there exists $\left(x_{1}^{*}, \ldots, x_{n}^{*}\right) \in \partial_{x_{1}} J_{1}(\bar{x}) \times \ldots \times \partial_{x_{n}} J_{n}(\bar{x})$ satisfying

$$
\left\{\begin{array}{c}
<x_{1}^{*}, x_{1}-\bar{x}_{1}>\geq 0, \text { for all } x_{1} \in K_{1}\left(\bar{x}_{-1}\right) \\
\cdot \\
\cdot \\
<x_{n}^{*}, x_{n}-\bar{x}_{n}>\geq 0, \text { for all } x_{n} \in K_{n}\left(\bar{x}_{-n}\right),
\end{array}\right.
$$

where $\partial_{x_{i}} J_{i}$ is the subdifferential of $J_{i}\left(., x_{-i}\right)$ in the sense of convex analysis, for all $i=1, \ldots, n$. To continue our treatment, consider the set-valued operator given by, for any $x \in X$,

$$
T(x)=\partial_{x_{1}} J_{1}(x) \times \ldots \times \partial_{x_{n}} J_{n}(x)
$$


and define the constraints map by

$$
\mathbb{K}(x)=\left\{y \in X \mid y_{i} \in K_{i}\left(x_{-i}\right), \forall i=1,2, \ldots, n\right\} .
$$

According to these objects, a Nash equilibrium point of the game $\left(X_{i}, J_{i}, K_{i}\right)_{1 \leq i \leq n}$ is consequently converted into the following generalized quasi-variational inequality: Find $\bar{x} \in X$ with $\bar{x} \in \mathbb{K}(\bar{x})$ such that there exists $x^{*} \in T(\bar{x})$ satisfying

$$
\left\langle x^{*}, x-\bar{x}\right\rangle \geq 0, \forall x \in \mathbb{K}(\bar{x}) .
$$

Definition 5.2. Let $\alpha>0$. A point $\bar{x} \in X$ is be said a weak $\alpha$-approximate Social Nash Equilibrium point for the game $\left(X_{i}, J_{i}, K_{i}\right)_{1 \leq i \leq n}$ if and only if

$$
\bar{x} \in \mathbb{K}(\bar{x}) \text { and there exists } x^{*} \in T(\bar{x})
$$

such that

$$
\left\langle x^{*}, x-\bar{x}\right\rangle \geq-\alpha\left\|x^{*}\right\|, \forall x \in \mathbb{K}(\bar{x}) .
$$

Let us state our result for this problem as follows.

Theorem 5.1. Let $X$ be a finite-dimensional Euclidian space. Suppose that, for all $i=1, \ldots n$, the function $J_{i}$ is continuous and convex on $X, K_{i}\left(x_{-i}\right)$ is convex and compact. Then, for every $\alpha>0$, the game $\left(X_{i}, J_{i}, K_{i}\right)_{1 \leq i \leq n}$ admits a weak $\alpha$-approximate Social Nash Equilibrium point.

Proof. The idea is to apply Theorem 4.1 with a given cone $C$ in $X$ with a nonempty interior and a compact $\mathscr{K}$ of $X$ such that $\mathbb{K}(x) \subset \mathscr{K}$, for all $x \in X$. Since, for each $i, K_{i}\left(x_{-i}\right)$ is convex and compact, then $\mathbb{K}(x)$ is convex and compact. Moreover, $T(x)$ is convex (as a product of convex sets). On the other hand, given that $J_{i}$ is continuous on $X$, for all $i=1, \ldots, n$, from [18, Proposition 1.11 ], it follows that $\partial_{x_{i}} J_{i}(x)$ is weak *-compact, for all $i=1, \ldots, n$. Then, $\partial_{x_{i}} J_{i}(x)$ is compact, for all $i=1, \ldots, n$, because $X$ is finite-dimensional. Therefore, $T(x)$ is a compact. Hence, by Theorem 4.1, the Social Nash Equilibrium problem $\left(X_{i}, J_{i}, K_{i}\right)_{1 \leq i \leq n}$ admits a weak $\alpha$-approximate Social Nash Equilibrium point, for every $\alpha>0$.

Remark 5.1. If, in addition to the assumptions of Theorem 5.1, the map $\mathbb{K}$ is Hausdorff continuous and the operator $T$ verifies the regularity condition $(\mathbb{H})$, then it follows from Theorem 4.2 that the game $\left(X_{i}, J_{i}, K_{i}\right)_{1 \leq i \leq n}$ admits an exact solution obtained as a limit of a sequence of weak approximate solutions to this problem.

\section{Conclusion}

In this paper, we considered a new concept of weak solutions to quasi-variational inequalities on the basis of lower semicontinuous regularizing maps. This type of auxiliary solutions works well for the convergence to exact solutions under an additional regularity conditions. The key idea is to regularize the closed convex hull of an auxiliary solution map obtained by a slight modification of the one considered in [2]. Our result for problem (QVI) was motivated by some examples and mainly by the application to Nash equilibrium problems.

Finally, we would like to mention the following points.

- For the purpose of the applications, it is worth determining conditions that ensure the regularity property $(\mathbb{H})$ for the cases when $T$ is the subdifferential of a convex function and Clarke subdifferential of a locally Lipschitz function respectively. 
- In the further, it would be interesting to analyze the corresponding weak minima (in the sense discussed in (3) of Examples 2.6 above) generated by our weak format of variational inequalities. It would be also interesting to envisage and examine applications of weak solutions to numerical optimization problems.

- We also believe that a parallel treatment of quasi-variational inclusions could be of a particular relevance. More precisely, for a locally Lipschitz function $J: X \longrightarrow \mathbb{R}$ and given maps $A: X \rightrightarrows X^{*}, \mathbb{K}: X \rightrightarrows X$, the problem: Find $u \in X$ such that $u \in \mathbb{K}(u)$ and

$$
0 \in A(u)+\partial^{c} J(u),
$$

where $\partial^{c} J$ is the Clarke subdifferential of $J$, deserves some attention as it encompasses other mathematical models such as quasi-hemivariational inequalities, quasioptimisation problems, quasiconvex programming and nonconvex and nonsmooth optimization.

- Regarding Nash games, in [19] and [20], the authors investigated the existence of projected solutions and stability analysis, respectively. Then, further questions that may be addressed concern the comparison of the different categories of solutions and their stability analysis in the nondifferentiable setting.

\section{Acknowledgments}

The authors wish to thank the Editor-in-Chief, and the anonymous referee for their useful comments and suggestions which helped to improve this paper.

\section{REFERENCES}

[1] M. Ait Mansour, M. Durea, M. Théra, A lower semicontinuous regularization for set-valued maps and its applications, J. Conv. Anal. 15 (2008), 473-484.

[2] M. Ait Mansour, J. Lahrache, N. Ziane, $\alpha$-strong approximate solutions to quasi-variational inequalities, Le Matematiche. LXXIII (2018) 115-125.

[3] J-L. Joly, U. Mosco, A propos de l'existence et de la régularité des solutions de certaines inéquations quasivariationnelles, J. Funct. Anal. 34 (1979), 107-137.

[4] D. Aussel, J. Cotrina, Quasimonotone Quasivariational inequalities: Existence Results and Applications, J. Optim. Theory. Appl. 158 (2013) 637-652.

[5] B.S. Mordukhovich, Variational Analysis and Generalized Differentiation. I: Basic Theory, Springer, 2006.

[6] G. Beer, Topologies on Closed and Closed Convex Sets, Kluwer Academic Publishers, Dordrecht, 1993.

[7] A. Göpfert, H. Riahi, C. Tammer, C. Zălinescu, Variational Methods in partially ordered Spaces, SpringerVerlag, New York, 2003.

[8] D. Aussel, J. Cotrina, Stability of quasimonotone variational inequalities under sign-continuity, J. Optim. Theory. Appl. 158 (2013), 653-667.

[9] B. Ricceri, Un theéoreme d'existence pour les inéquations variationnelles, Comptes Rendus Acad. Sci. Paris, Série I 301 (1985), 885-888.

[10] P. Cubiotti, N.D. Yen, A result related to Ricceri's conjecture on generalized quasi-variational inequalities, Arch. Math 69 (1997), 507-514.

[11] G. Debreu, A social equilibrium existence theorem, Proc. Nat. Acad. Sci., USA, 38 (1952), 886-893.

[12] T. Ichiichi, Game Theory for Economic Analysis, Academic Press, New York, 1983.

[13] J.F. Nash, Equilibrium points in n-person games, Proc. Nat. Acad. Sci. USA, 36 (1950), 48-49.

[14] A. Bensoussan, Points de Nash dans le cas de fonctionnelles quadratiques et jeux differentials linéaires a $\mathrm{N}$ personnes, SIAM J. Control 12 (1974), 460-499. 
[15] E. Cavazzuti, M. Pappalardo, M. Passacantando, Nash equilibria, variational inequalities and dynamical systems, J. Optim. Theory. Appl. 114 (2002), 491-506.

[16] J. Morgan, M. Romaniello, Generalized quasi-variational inequalities and duality, J. Inequal. Pure Appl. Math. 4 (2003), 28.

[17] P.T. Harker, Generalized Nash games and quasi-variational inequalities, Euro. J. Oper. Res. 54 (1991), 81-94.

[18] R. Phelps, Convex functions, monotone operators, and differentiability, Lecture Notes in Mathematics 1364, Berlin-Heidelberg-New York, 1989.

[19] D. Aussel, A. Sultana, V. Vetrivel, On the existence of projected solutions of quasi-variational inequalities and generalized Nash equilibrium problems, J. Optim. Theory Appl. 170 (2016), 818-837.

[20] R. T. Rockafellar, Variational Analysis of Nash Equilibrium, Vietnam J. Math. 46 (2018), 73-85. 